\title{
Manejo diagnóstico y terapéutico de un quiste de vallécula en un lactante
}

\section{Diagnostic and therapeutic management of a vallecular cyst in an infant}

\author{
Mikel García M. ${ }^{1}$, Ana Moreno V. ${ }^{1}$, Ignacio Toribio R. ${ }^{1}$, Tomás Mogollón C. ${ }^{1}$, Carlos Montero G. ${ }^{1}$
}

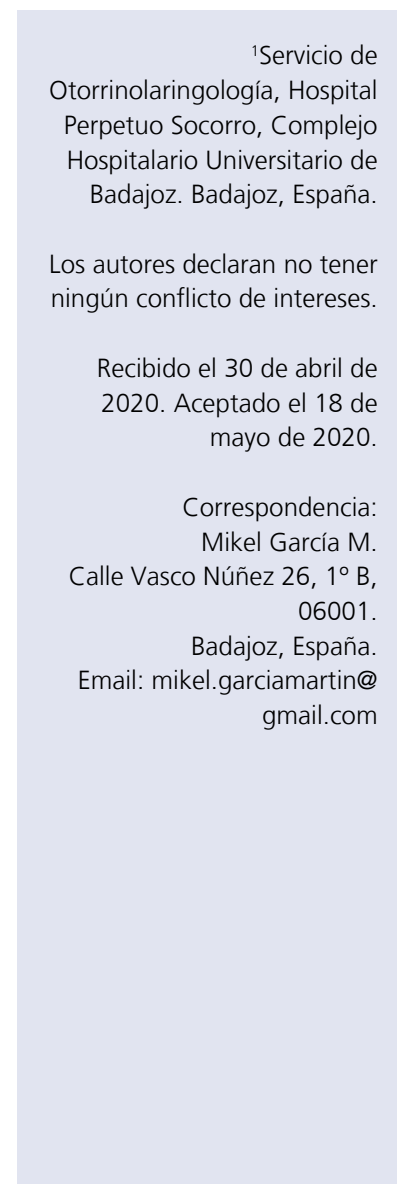

\section{Resumen}

Los quistes laríngeos, y en particular los quistes de vallécula, son una entidad infrecuente en la práctica clínica habitual. Sin embargo, su localización y aparición en neonatos y lactantes, pueden suponer una causa reconocida de estridor y obstrucción de vía aérea superior que, si no es diagnosticada y tratada de forma precoz, puede tener consecuencias fatales. Presentamos el caso de un lactante que presenta estridor inspiratorio. Se realiza una nasofibrolaringoscopía observándose una formación quística en la vallécula, y una ecografía cervical que muestra dicha formación quística sugerente de un quiste del conducto tirogloso como primera posibilidad etiológica. Ante estos hallazgos, se solicitan pruebas tiroideas y una gammagrafía que son normales, por lo que se decide intervenir al paciente bajo laringoscopia en suspensión, realizando una marsupialización del quiste, sin complicaciones posteriores. El análisis histopatológico posterior confirmó el diagnóstico de un quiste de vallécula. Se debe considerar esta patología en los casos de estridor inspiratorio en lactantes ya que, aunque es infrecuente, su diagnóstico precoz y tratamiento adecuado son determinantes.

Palabras clave: Quiste laríngeo, quiste de vallécula, estridor, laringoscopia, marsupialización.

\begin{abstract}
Laryngeal cysts, and especially vallecular cysts, are a rare entity in everyday clinical practice. Nevertheless, their location and the fact that they appear in newborns and infants, must be recognized as a cause of stridor and upper airway obstruction, since their misdiagnosis and late treatment can have fatal consequences. We present the case of an infant with inspiratory stridor. We performed a nasofibrolaryngoscopy where a vallecular cystic lesion was observed, and a neck ultrasound showed a cyst, described as a thyroglossal duct cyst, as the main etiology. Thyroid function tests and a gammagraphy, were both normal, hence the patient underwent a suspension microlaryngoscopy and marsupialization of the cyst, without further complications. The histopathology confirmed the diagnosis of a vallecular cyst. This entity must be considered in infants with inspiratory stridor. Although it is a rare lesion, its early diagnosis and adequate management is crucial.
\end{abstract}

Keywords: Laryngeal cyst, vallecular cyst, stridor, laryngoscopy, marsupialization.

\section{Caso Clínico}

Varón lactante de 2 meses de edad nacido de parto gemelar por cesárea a las 36 semanas, sin comorbilidad añadida, es llevado al servicio de urgencia por presentar estridor inspiratorio, con tiraje acompañante, cada vez más acentuado desde hace 3 semanas.
Los padres refieren una disminución de ingesta por fatiga y palidez con las tomas de pecho materno, pero con adecuada ganancia ponderal. El paciente no presentaba cianosis, hipotonía, disfonía ni otra sintomatología de la vía aérea. En la exploración física, presenta saturación de oxígeno al 100\%, palidez cutánea, polipnea, estridor inspiratorio, hipo- 
fonesis generalizada con ruidos altos, pectus excavatum, y retracción xifoidea.

Se solicita una evaluación por el equipo de otorrinolaringología, y se realiza una nasofibrolaringoscopía con la cual se evidencia una formación de aspecto quístico en la vallécula derecha de aproximadamente $1,5 \mathrm{~cm}$. Ante estos hallazgos, se decide solicitar una ecografía cervical en la que se objetiva una lesión de aspecto quístico en la línea media supraglótica, que mide aproximadamente $1,3 \times 1,3 \times 1,5 \mathrm{~cm}$, sin contenido ecogénico ni captaciones. Doppler color, sugestivo de quiste del conducto tirogloso o tiroides lingual, como primera posibilidad etiológica (Figura 1).

Se solicitan pruebas tiroideas que no muestran alteraciones, y una gammagrafía tiroidea, en la que se observa actividad del trazador en ambos lechos tiroideos, compatible con una glándula tiroidea funcionante y normoposicionada, y no se aprecian depósitos focales en área sublingual ni en tercio superior del cuello que sugieran la presencia de tejido tiroideo ectópico funcionante.

Dado los hallazgos de las pruebas complementarias, se decide realizar una intervención quirúrgica bajo anestesia general. Se realiza una microcirugía laríngea con laringoscopía en suspensión, y con óptica de $0^{\circ}$ se visualiza un quiste mucoso en vallécula que se marsupializa mediante exéresis de pared mucosa con bisturí frío (Figura 2).

El análisis histológico de la lesión muestra una formación quística tapizada por epitelio escamoso no queratinizante compatible con un quiste laríngeo. El paciente pasa intubado a la unidad de cuidados intensivos pediátricos, se extuba a las 24 horas y se mantiene en dicha unidad 48 horas con buena evolución. A los 5 días se realiza una nueva nasofibrolaringoscopía en la que no se observa recidiva ni ninguna otra complicación. El paciente es dado de alta a la semana, manteniéndose asintomático y sin evidencias de recidiva hasta última revisión al año y medio.

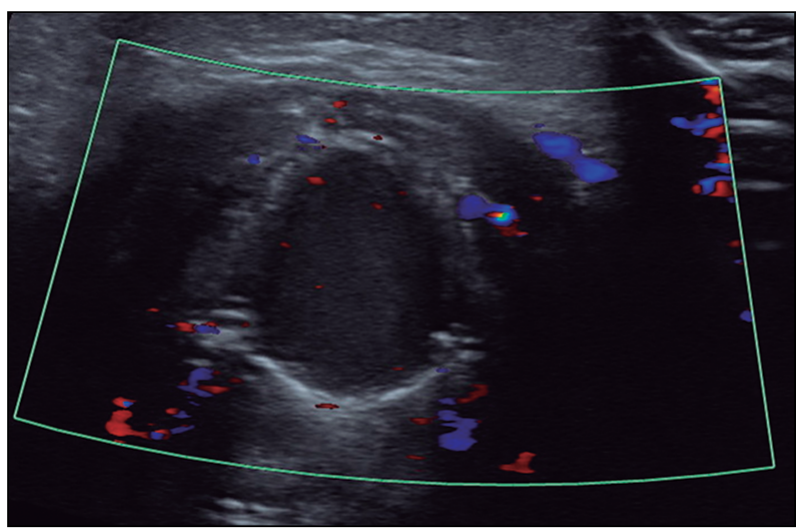

Figura 1. Ecografía cervical. Doppler color que muestra lesión quística sin captaciones significativas.

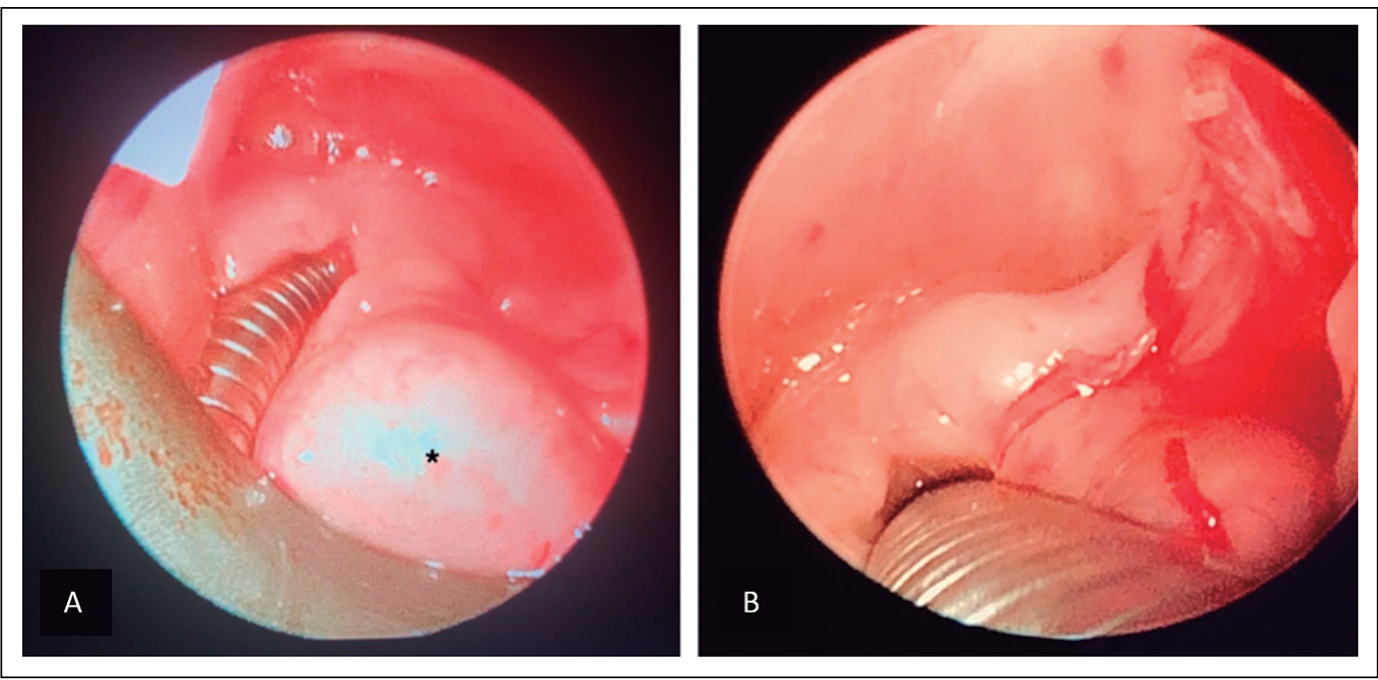

Figura 2. (A) Visualización intraoperatoria del quiste de vallécula derecha (*) mediante laringoscopia suspendida previo a marsupialización. (B) Visión intraoperatoria de zona intervenida tras marsupialización del quiste. 


\section{Discusión}

El primer caso de quiste de vallécula fue descrito por Abercrombie en $1881^{1}$. Los quistes laríngeos son una entidad infrecuente, estimándose su incidencia en 1,87-3,49 por cada 100.000 nacidos vivos. Entre ellos, los quistes de vallécula suponen entorno al 10,5\% del total ${ }^{2,3}$. Estas malformaciones laríngeas pueden ser de dos tipos: quistes saculares, formados por una dilatación con contenido mucoso del sáculo del ventrículo laríngeo; o quistes ductales, más frecuentes, y debidos a una obstrucción en la salida de glándulas mucosas, aunque también pueden tener un origen traumático. Los quistes de vallécula son generalmente de tipo ductal ${ }^{4,5}$. Histológicamente, están conformados por un epitelio escamoso no queratinizado y mucosa glandular $^{2-4}$. Se trata de una entidad rara, pero es una causa conocida de estridor en niños ${ }^{2-8}$.

Esta patología puede ser asintomática en algunos casos y detectarse en edad adulta, si bien en neonatos y lactantes es frecuente la aparición de dificultad para las tomas y disfagia, vómitos posprandiales, tos, episodios de apnea, estridor inspiratorio, obstrucción de la vía aérea superior, o incluso muerte. Estos síntomas pueden estar presentes desde el nacimiento, aunque es más frecuente que se agudicen con la agitación del niño, y se vayan acentuando conforme el quiste va creciendo a lo largo de semanas o meses ${ }^{2-8}$.

Es fundamental realizar un diagnóstico diferencial con otras entidades que producen similar sintomatología como pueden ser tiroides lingual y quiste del conducto tirogloso, como en nuestro caso, pero también con un quiste dermoide, linfangioma, teratoma, hamartoma, laringocele, lipoma y hemangioma ${ }^{4,5,8,9}$.

La forma y el tiempo para poder diagnosticarlos varía en función de su tamaño. Algunos autores hablan de la posibilidad de detección prenatal si este ya tiene un tamaño valorable para entonces, gracias a la ecografía a partir de la semana 25 de gestación ${ }^{2,7,8}$. Sin embargo, lo más habitual es su detección tras el parto, cuando el quiste empieza a crecer y el niño comienza con sintomatología. Numerosos textos recomiendan la nasofibrolaringoscopía como primera prueba diagnóstica, como realizamos en nuestro centro, al tratarse de una técnica sencilla, rápida y poco invasiva ${ }^{2-8}$, aunque no siempre es posible llegar a identificar el quiste o su localización exacta ${ }^{2,3,5}$.

Se ha mostrado útil la radiografía simple lateral de cuello como primera prueba de imagen $^{2-7}$, sin embargo, puede resultar difícil de interpretar, $y$ los quistes pequeños pueden pasar desapercibidos ${ }^{3,6,7}$. También la ecografía es una técnica ampliamente utilizada por su rapidez y mínima invasión, porque permite distinguir entre masas sólidas y quísticas, y valorar la presencia de un tiroides de aspecto y localización normales ${ }^{2,3,7}$, aunque en nuestro caso esto último no se pudo lograr. La tomografía computarizada (TC) y la resonancia magnética son las pruebas radiológicas de elección, permiten definir el tamaño, la localización, la procedencia y las relaciones del quiste con estructuras vecinas $^{2,3,7}$, pero dado que precisan sedación y con el posible compromiso de la vía aérea ${ }^{2,3,5,8}$, en nuestro caso preferimos iniciar estudio con ecografía cervical. Además, la TC supone exponer al niño a altas dosis de radiación ${ }^{8}$. Ante la duda diagnóstica, como en el caso expuesto con un posible tiroides lingual, se deben solicitar pruebas tiroideas y gammagrafía tiroidea para determinar la funcionalidad de la glándula tiroides, y así descartar que se trate de un quiste del conducto tirogloso o tiroides ectópico ${ }^{3,9}$. Pese a todo ello, la técnica de elección para el diagnóstico, y útil en el tratamiento, es la laringoscopía directa ${ }^{2-9}$.

El tratamiento de esta patología es quirúrgico. La punción aspirativa presenta altas tasas de recurrencia, y debería usarse solo en casos urgentes o como ayuda en la intubación ${ }^{3,5-7}$. El tratamiento debe realizarse bajo visión directa con endoscopia o laringoscopia suspendida ${ }^{2,10}$. Algunos autores defienden la necesidad de exéresis completa de la pared del quiste ${ }^{4,10}$, sin embargo, algunas veces esto es difícil de lograr y solo es posible por vía externa, lo que limita su uso ${ }^{2,3,8}$. Además, la mayor parte de autores consideran correcta la realización de una marsupialización con técnica fría o láser $\mathrm{CO}_{2}$, sin encontrar grandes diferencias con respecto a la exéresis completa ${ }^{2,3,5,6,8}$.

\section{Conclusión}

Los quistes de la vallécula son una patología infrecuente. Poseen un amplio rango de sintomatología que puede ir desde asintomáticos 
hasta estridor inspiratorio, obstrucción de la vía aérea y muerte, sobre todo en lactantes y neonatos. Por ello, ante la presencia de esta sintomatología, deben tenerse en cuenta los quistes laríngeos, ya que su diagnóstico temprano, realizando un correcto diagnóstico diferencial con otras entidades que producen similar sintomatología, y su tratamiento, mediante marsupialización o exéresis completa, son claves a la hora de prevenir un desenlace no deseado.

\section{Bibliografía}

1. Abercrombie J. Congenital cyst in larynx. Trans Pathol Soc London 1881;32:33-34.

2. Li Y, Irace AL, Dombrowski ND, Pérez-Atayde AR, Robson CD, Rahbar R. Vallecular cyst in the pediatric population: Evaluation and management. Int J Pediatr Otorhinolaryngol. 2018;113:198-203. doi: 10.1016/j. ijporl.2018.07.040.

3. Suzuki J, Hashimoto S, Watanabe K, Takahashi $\mathrm{K}$. Congenital vallecular cyst in an infant: case report and review of 52 recent cases. J Laryngol Otol. 2011;125:1199-1203. doi: 10.1017/ S0022215111001368.

4. Rodríguez H, Cuestas G, Zanetta A. Quistes congénitos de laringe: saculares y ductales. Rev FASO. 2013;20:53-58.

5. Leibowitz JM, Smith LP, Cohen MA, Dunham BP, Guttenberg M, Elden LM. Diagnosis and treatment of pediatric vallecular cysts and pseudocysts. Int J Pediatr Otorhinolaryngol. 2011;75:899-904. doi: 10.1016/j.ijporl.2011.03.032.

6. Sands NB, Anand SM, Manoukian JJ. Series of congenital vallecular cysts: a rare yet potentially fatal cause of upper airway obstruction and failure to thrive in the newborn. J Otolaryngol Head Neck Surg. 2009;38(1):6-10.

7. Breysem L, Goosens V, Poorten VV, Vanhole C, Proesmans M, Smet M. Vallecular cyst as a cause of congenital stridor: report of five patients. Pediatr Radiol. 2009;39:828-831. doi: 10.1007/s00247-0091299-1.

8. Osorio J, Cancino M, Arregui R, Suazo A. Quiste de vallécula como causa de estridor congénito. Reporte de un caso y revisión de la literatura. Rev Otorrinolaringol Cir Cabeza Cuello. 2016;76:236-241.

9. Gogia S, Agarwal SK, Agarwal A. Vallecular Cyst in Neonates: Case Series-A Clinicosurgical Insight. Case Rep Otolaryngol. 2014;2014:764860. doi: 10.1155/2014/764860.

10. Chen EY, Lim J, Boss EF, et al. Transoral approach for direct and complete excision of vallecular cysts in children. Int J Pediatr Otorhinolaryngol. 2011;75(9):1147-1151. doi: 10.1016/j. ijporl.2011.06.007. 